\title{
The pupillary response to light in Type 1 (insulin-dependent) diabetes
}

\author{
Á. B. Hreidarsson* and H.J.G.Gundersen \\ Second University Clinic of Internal Medicine, Kommunehospitalet, Aarhus, Denmark
}

\begin{abstract}
Summary. The pupillary response to light was examined by infrared television-videopupillography in 93 Type 1 (insulin-dependent) diabetic patients (aged 25-42 years, duration of diabetes $0-32$ years), and 37 control subjects (aged 26-41 years) with techniques ensuring equality of stimulus and retinal sensitivity, and allowing a detailed computerized calculation of the various parameters of the response. There was no difference in latency time or constriction time between diabetic patients and control subjects. The diabetic patients had a smaller initial pupil size $(p=0.012)$ and a smaller response amplitude $(p<0.001)$ than the control subjects, and these two parameters were correlated to each other $(r=0.49, p<0.000001)$ and inversely correlated to the duration of diabetes $(r=-0.26$, $p=0.013$ and $r=-0.29, p=0.0051$, respectively). As a group, the diabetic patients had a relative response amplitude that was similar to that of the control subjects. However, more detailed analysis showed that the diabetic patients with pupil size in the normal range had a small, but significant, reduction in relative response amplitude $(p=0.0021)$. The maximal velocities of constriction and re-dilatation were reduced in the diabetic patients ( $p<0.001$ in either case), but both parameters
\end{abstract}

were intimately correlated to the response amplitude $(r=0.91$, $p<0.000001$, and $r=0.79, p<0.000001$, respectively), and this relationship was identical in the control subjects. Analysis of velocity-size plot for long-term diabetic patients showed no systematic deviation from that of non-diabetic subjects, indicating unaltered dynamic properties of the small pupil within the altered dynamic range in diabetic patients. The foremost change in the pupils of long-term diabetic patients is a reduction in size. Since normal iris dynamics are preserved, the small pupil must be due to loss of sympathetic tone. When diabetic patients with a normal or near-normal sensory pathway are stimulated by light, they have a normal latency time and response parameters that are normal for the size of their pupil. The only exception seems to be a minor reduction in response amplitude, possibly indicating damage to the efferent, parasympathetic pathways, as is known to develop in other organs.

Key words: Pupil, light response, Type 1 diabetes, autonomic neuropathy, pupillography.
Abnormal pupillary response to light in a diabetic patient was reported by Pryce [1] almost a century ago. Several cases have been reported subsequently $[2,3]$ and investigations of large groups of diabetic patients have revealed abnormal responses in a number of patients [4], especially in those with diabetic neuropathy [5-7]. Typically, a small pupil with a sluggish reaction to light was reported, but often with a preserved reaction to accommodation, some of these being termed "Argyll Robertson pupils" [7].

While these early studies relied on the clinical evaluation of the pupillary response, subsequent workers, benefiting from the advent of pupillographic tech-

* Present address: Medical Department, Landspítalinn, University Hospital, Reykjavík, Iceland niques, have studied the response more accurately. Ohrt [8] examined 85 long-term diabetic patients by a cinematographic technique, and found no changes in the initial diameter, latency time, rate of contraction or amplitude of the response. However, in about one-third of the patients, an abnormally prolonged dilatation phase was observed, possibly indicating sympathetic nervous dysfunction. Friedman et al. [9], employing electronic infrared pupillography, examined 22 Type 2 (non-insulin-dependent) diabetic patients (aged 39-49 years) and found prolonged latency time and a number of cases with shallow response. On the contrary, Gliem [10], with a similar technique, found a shorter latency time of the light response in diabetic patients with short duration of disease, and no prolongation of the latency period in patients with longer duration. The resting pupil size, the response amplitude, and the constriction velocity were 


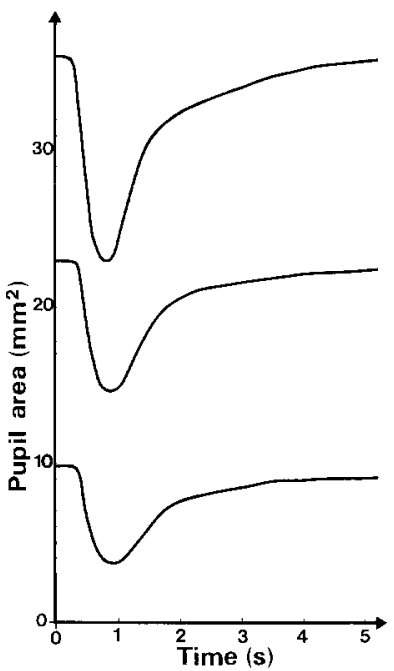

Fig.1. Computer drawing of the pupil response to a 256-ms long, $25 \mathrm{ulm}$ light stimulus. Upper curve: control subject; middle curve: long-term diabetic patient; lower curve: the long-term diabetic patient with the smallest pupil. Each curve is the mean of at least 10 consecucutive responses

diminished in the diabetic patients, but the constriction time was unchanged.

In an earlier infrared television-pupillographic study of 109 Type 1 diabetic patients and 39 control subjects, we demonstrated that the size of the pupil is normal shortly after onset of the disease, but it decreases gradually with increasing duration of the disease. Thus, after 25 years of diabetes, on average, the pupil is only twothirds the size of normal [11]. This is interesting in itself, but becomes of methodological importance if one wishes to examine the reaction to light of the pupil in diabetic patients.

In an earlier study, it was shown that the reaction to light of the pupil was reduced in diabetic patients, but if expressed as a fraction of the abnormally small pupil at rest, it was normal [12]. However, the study included only a small number of patients.

The aim of the present study was to try to solve some of the controversies regarding the pupillary response in Type 1 diabetes by examining a larger group of diabetic patients by techniques that ensure the equality of the light stimulus and allow detailed computerized calculation of the various parameters of the response.

\section{Subjects and methods}

\section{Subjects}

The 93 Type 1 diabetic patients ( 58 males, 35 females) studied had a mean age of 34 years (range 25-42 years) and a mean duration of diabetes of 15 years (range $0-32$ years). Fifty had long-term diabetes with duration $\geq 15$ years. The 37 healthy control subjects $(15$ males, $22 \mathrm{fe}$ males) studied had an average age of 33 years (range 26-41 years). There was no relationship between age and duration of diabetes among the diabetic patients. Informed consent was obtained from all participants in the study, which was performed in accordance with the principles of the Declaration of Helsinki. 
Table 2. Light-response of the pupil to a $25 \mu \mathrm{lm}$ square-pulse of $256 \mathrm{~ms}$ duration

\begin{tabular}{lllllllll}
\hline & $\begin{array}{l}\text { Initial } \\
\text { size }\left(\mathrm{mm}^{2}\right)\end{array}$ & $\begin{array}{l}\text { Latency } \\
\text { time }(\mathrm{ms})\end{array}$ & $\begin{array}{l}\text { Constriction } \\
\text { time }(\mathrm{ms})\end{array}$ & $\begin{array}{l}\text { Response } \\
\text { amplitude } \\
\left(\mathrm{mm}^{2}\right)\end{array}$ & $\begin{array}{l}\text { Relative } \\
\text { response } \\
\text { amplitude } \\
(\%)\end{array}$ & $\begin{array}{l}\text { Maximal } \\
\text { constriction } \\
\text { velocity } \\
\left(\mathrm{mm}^{2} / \mathrm{s}\right)\end{array}$ & $\begin{array}{l}\text { Maximal } \\
\text { redilatation } \\
\text { velocity } \\
\left(\mathrm{mm}^{2} / \mathrm{s}\right)\end{array}$ & $\begin{array}{l}75 \% \text { re- } \\
\text { dilatation } \\
\text { time }(\mathrm{s})\end{array}$ \\
\hline $\begin{array}{l}\text { Diabetic } \\
\text { patients } \\
\begin{array}{l}\text { Control } \\
\text { subjects }\end{array}\end{array}$ & $27.5 \pm 7.5$ & $236 \pm 30$ & $821 \pm 61$ & $9.7 \pm 2.7$ & $35.8 \pm 9.2$ & $36.4 \pm 9.5$ & $12.3 \pm 3.2$ & $\begin{array}{l}\text { Relative } \\
\text { redilatation } \\
\text { residue }(\%)\end{array}$ \\
\hline$p$ & $31.5 \pm 5.8$ & $231 \pm 33$ & $828 \pm 53$ & $11.8 \pm 2.6$ & $38.6 \pm 9.7$ & $42.8 \pm 9.2$ & $14.4 \pm 2.6$ & $2.72 \pm 0.68$ \\
\hline
\end{tabular}

Results expressed as mean $\pm \mathrm{SD}$
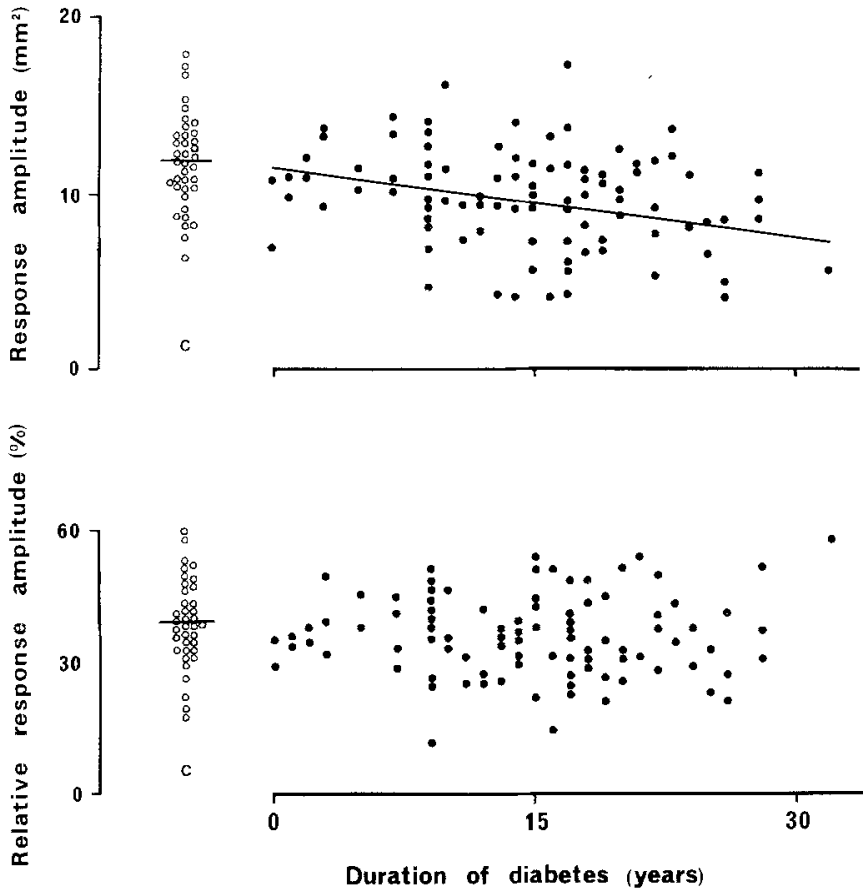

Fig. 2. Relationships between the duration of diabetes and the absolute response amplitude and relative response amplitude in 93 diabetic patients (-). The regression line for the response amplitude is shown. Values for the control subjects $(O)$ are shown on the left

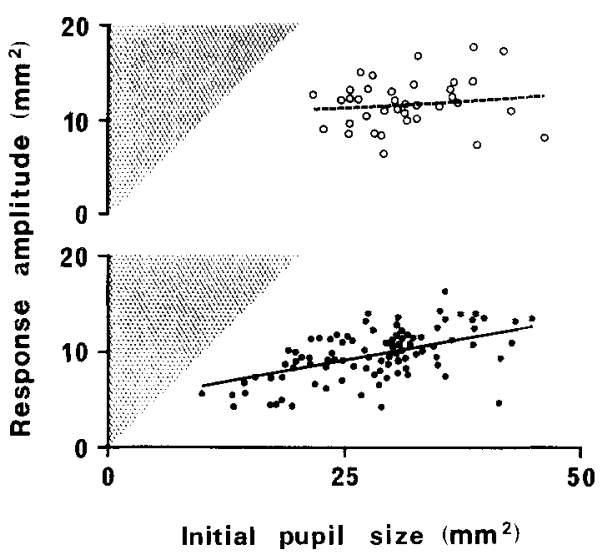

Fig.3. Relationship between absolute response amplitude and initial pupil size in the 37 control subjects (O) and 93 diabetic patients (O). The shading covers the area within which this relationship cannot exist. Regression lines are shown (diabetic patients: $r=0.50, p<$ 0.000001 ; the relationship is not significant in the control subjects)
Pupil size in darkness and its relationship to other long-term diabetic manifestations in virtually the same groups of subjects have been reported elsewhere [11].

Clinical data and diabetic complications are summarized in Table 1: diabetic retinopathy was present in 36 patients (39\%), six of these having proliferative changes. Biomicroscopic evidence of minor diabetic iridopathy as defined by Ohrt [8] was present in 10 of the 66 patients $(15 \%)$ who underwent slit lamp examination. Two had a minor degree of rubeosis iridis. Persistent proteinuria was present in six $(7 \%)$, and 29 of the 87 patients examined (33\%) had peripheral neuropathy, as shown by an abnormally high vibratory perception threshold [13].

Criteria for inclusion in the study were: (1) visual acuity of $6 / 9$ or better, (2) absence of visible retinopathy (except for three microaneurysms or less) in the area stimulated by the light pulse (see below), (3) no myopia or hypermetropia $>2$ dioptres, (4) no visible cataracts. The mean \pm SD non-fasting blood glucose level at the time of the study was $10.9 \pm 4.1 \mathrm{mmol} / 1$. Patients with hypoglycaemia (blood glucose $<3.5 \mathrm{mmol} / \mathrm{l}$ ) or excessive hyperglycaemia (blood glucose $>$ $20 \mathrm{mmol} / \mathrm{l}$ ) were excluded. The mean daily insulin requirement was $47.4 \pm 17.6 \mathrm{IU}$. None of the diabetic patients received any drugs other than insulin and none of the control subjects were on drug treatment.

\section{Methods}

The light stimulus was presented to the right eye by a photostimulator (Hamamatsu TV Corporation, Hamamatsu, Japan) producing a beam of yellow-green light converging in the plane of the pupil to a diameter of $1 \mathrm{~mm}$, thus eliminating variation in light input due to variation in pupil size. At the retinal level, the light beam covered an area of approximately the size of the optic disc and located one papillary diameter above the optic disc. Examination took place in darkness, to which the subjects had adapted for $15 \mathrm{~min}$. A red fixation point, placed at an individually adjusted optically infinite distance, was used to prevent accommodation. Details on the technique have been published elsewhere [14].

Pupillography was performed with an infrared sensitive TV-camera (Iriscorder, Hamamatsu) which measured the area of the left pupil. After analogue-to-digital conversion (Schlumberger, Solarthron, A210, Munich, FRG), the measurements were handled off-line on a central computing facility (CDC Cyber 173, Regional EDP-Centre, University of Aarhus, Denmark.)

The light response was elicited with a square wave increase in the light intensity from 1 to $26 \mu$ Lumen for $256 \mathrm{~ms}$. This was repeated several times (usually more than 10 times) allowing at least $15 \mathrm{~s}$ between each light pulse. The responses of the consensually reacting left eye were recorded for $5 \mathrm{~s}$ each at a $100 \mathrm{~Hz}$ sampling rate. The same protocol was used with a stronger light pulse, intensity from 1 to $51 \mu \mathrm{lm}$ in 79 of the diabetic patients and 27 control subjects.

From the mean curve of the response the following parameters were obtained (Fig. 1): (1) Initial size $\left(\mathrm{mm}^{2}\right)$ : the size of the pupil before stimulation. The day-to-day intra-individual variation of this parameter was $6.9 \%$, coefficient of variation (CV) estimated from re-in- 

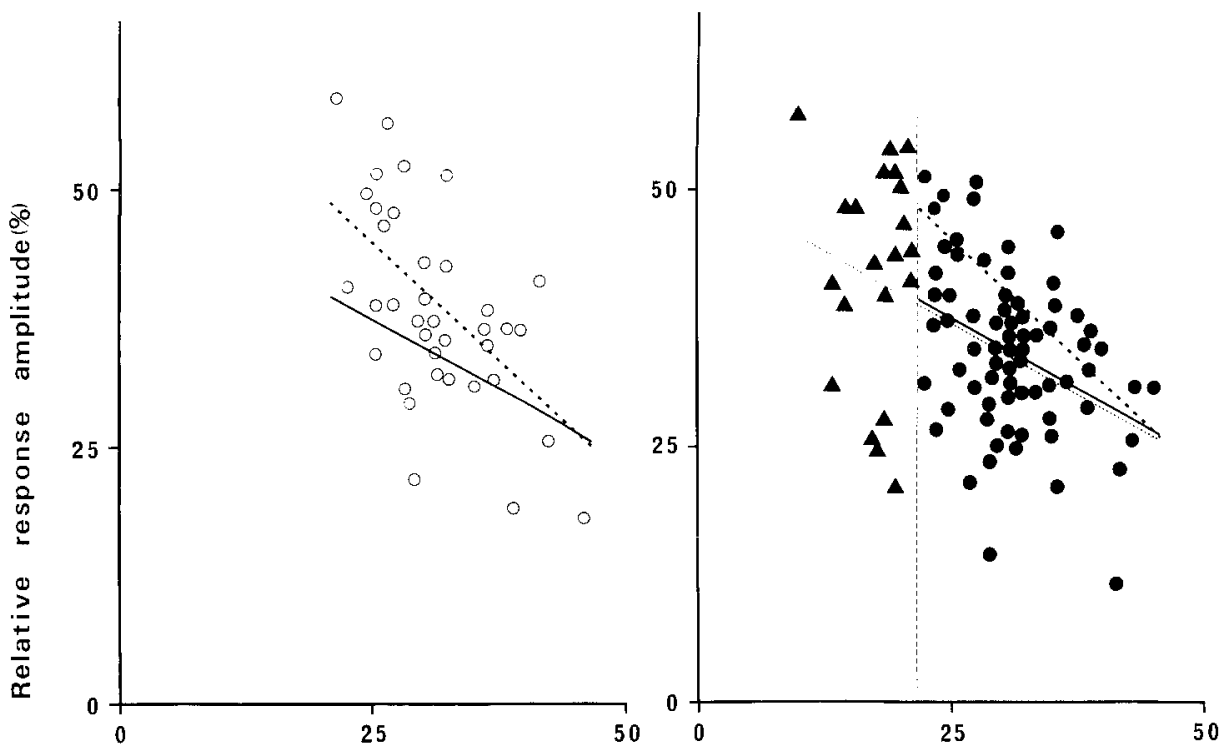

Fig.4. Relationship between relative response amplitude and initial pupil size in 37 control subjects (O), and 93 diabetic patients. The vertical line separates the values for pupil sizes below $(\boldsymbol{\Delta})$ and above ( ) the smallest pupil size in normal subjects. The regression line for the control subjects is dashed, that for the diabetic patients with pupil sizes above the smallest size in control subjects is fulldrawn, and that for the patients with pupil sizes below the smallest size in control subjects is dotted

Initial pupil size $\left(\mathrm{mm}^{2}\right)$
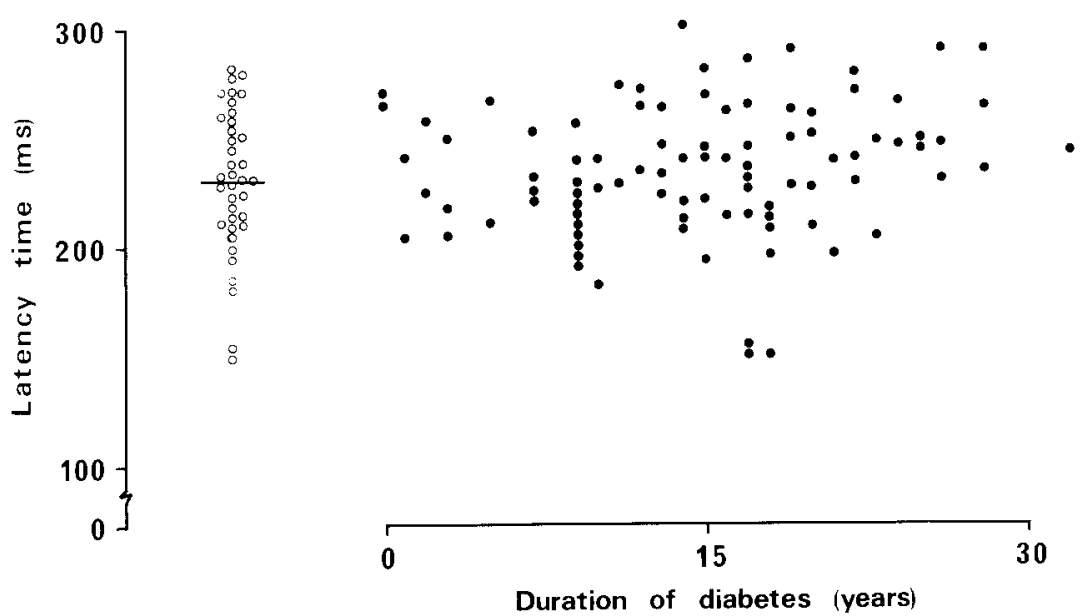

Fig.5. Relationship between latency time and duration of diabetes in 93 diabetic patients $(\bullet)$ and 37 control subjects $(O)$

vestigations $<1$ month apart. (2) Latency time (ms): the period from the initiation of the light pulse until $1 / 10$ of maximal constriction velocity was obtained (CV: $8.7 \%$ ). (The algorithm for calculating the latency time was constructed so as to exclude interference from spontaneous variations in pupil size during the latency period.) (3) Maximal constriction velocity $\left(\mathrm{mm}^{2} / \mathrm{s}\right)$ : the steepest slope of the declining part of the response curve (CV: $5.7 \%$ ). (4) Constriction time (ms): the period from the start of the light pulse until the minimal size was obtained (CV: 3.3\%). (5) Response amplitude $\left(\mathrm{mm}^{2}\right)$ : initial size minus minimal size (CV: 6.6\%). (6) Maximal redilatation velocity $\left(\mathrm{mm}^{2} / \mathrm{s}\right)$ : the steepest slope of the rising part of the response curve (CV: $8.2 \%)$. (7) Redilatation time (s): the period from the initiation of the light pulse until the pupil had regained $75 \%$ of its initial size (CV: 17.6\%). All these parameters were unequivocally defined in numerical algorithms and were calculated during the computer treatment of the responses.

The following relative parameters of the responses were also calculated: (1) The relative response amplitude: the response amplitude as a percentage of the initial pupil size (CV: $6.5 \%)$. (2) The relative redilatation residue: the difference between the initial pupil area and the area at the end of the 5-s period as a percentage of the amplitude of the response (CV: $31 \%)$.

In some patients, the simultaneous values of response velocity $\left(\mathrm{mm}^{2} / \mathrm{s}\right)$ and size $\left(\mathrm{mm}^{2}\right)$ during the responses to light were calculated according of the principles of Stark [15]. Examples of such a calculation are shown in Figure 7. To compare the shape of this velocity-size graph independent of its position and the enclosed area in the individual subjects, the graphs underwent two transformations during their computer treatment: (1) they were magnified so as to enclose the same area; (2) the graphs were translocated to a common origin.

\section{Statistical analysis}

For comparison between groups, Student's t-test was used, and for testing the strength of correlations, an analysis of the ordinary parametric least square regression was performed. A $5 \%$ limit of significance was used throughout the study and ' $p$ ' was two-tailed.

\section{Results}

The parameters of the pupil response to a $25 \mu \mathrm{lm}$ light stimulus in diabetic patients and control subjects are shown in Table 2. The diabetic patients had an $11 \%$ smaller initial pupil size $(p=0.012)$ and an $18 \%$ smaller response amplitude $(p<0.001)$. However, the relative response amplitude was not significantly smaller. The long-term diabetic patients (with duration of 15 years 


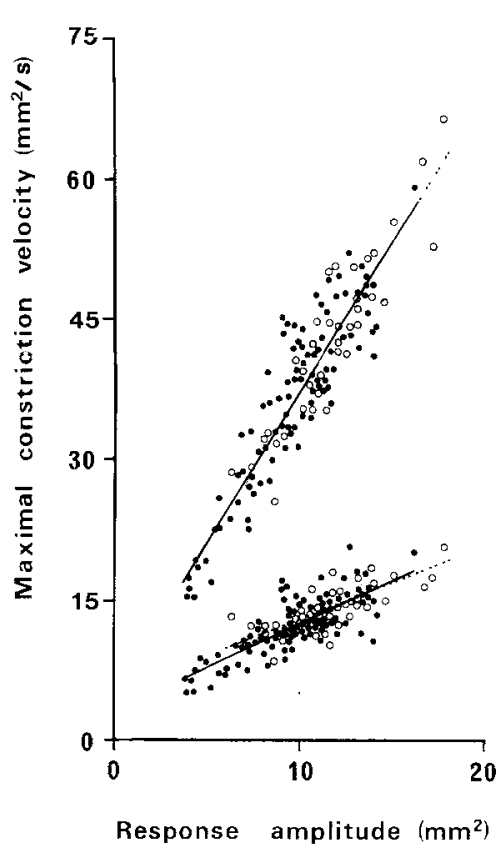

Fig. 6. Relationships between constriction velocity (upper-left part) and maximal redilatation velocity (lower-right part) and response amplitude in 93 diabetic patients (-) and 37 control subjects (O). The regression lines (diabetic patients: - $\longrightarrow$, control subjects: --..--) are identical in either case and are therefore superimposed

or more) had smaller pupils than those with duration < 15 years (mean $\pm \mathrm{SD}: 26.2 \pm 8.0$ versus $29.9 \pm 6.4 \mathrm{~mm}^{2}$; $p=0.020)$, and a smaller response amplitude $(9.0 \pm 2.6$ versus $10.4 \pm 2.7 \mathrm{~mm}^{2} ; p=0.013$ ), but there was no difference in the relative response amplitude between these two groups $(36.2 \pm 10.2$ versus $35.5 \pm 7.8 \%)$.

In the diabetic patients, both the initial pupil size and the response amplitude were inversely correlated to the duration of diabetes $(r=-0.26, p=0.013$ (data not shown), and $r=-0.29, p=0.0051$, respectively; Fig. 2). There was, however, no correlation between the relative response amplitude and the duration of diabetes (Fig. 2).

A positive correlation was obtained between initial pupil size and response amplitude in the diabetic patients $(r=0.50, p<0.000001)$, whereas there was none in the control group (Fig. 3). However, there was an inverse relationship between initial pupil size and relative response in control subjects $(r=-0.56, p<0.001$; Fig. 4) and among 72 diabetic patients ( $r=-0.39, p<$ 0.001 ) with an initial pupil size in the normal range $\left(21.7-45.9 \mathrm{~mm}^{2}\right)$. The slopes of the regression lines were similar, but the regression line of the diabetic patients was below that of the control subjects ( $p=0.0021$, with a mean displacement of $12 \%$ ). This means that for a given initial pupil size in the normal range, the diabetic patients had a smaller relative response than the control subjects. If the diabetic patients with initial pupil sizes below normal are included, the correlation is even more pronounced, but a direct comparison with the control subjects is then obviously not meaningful.
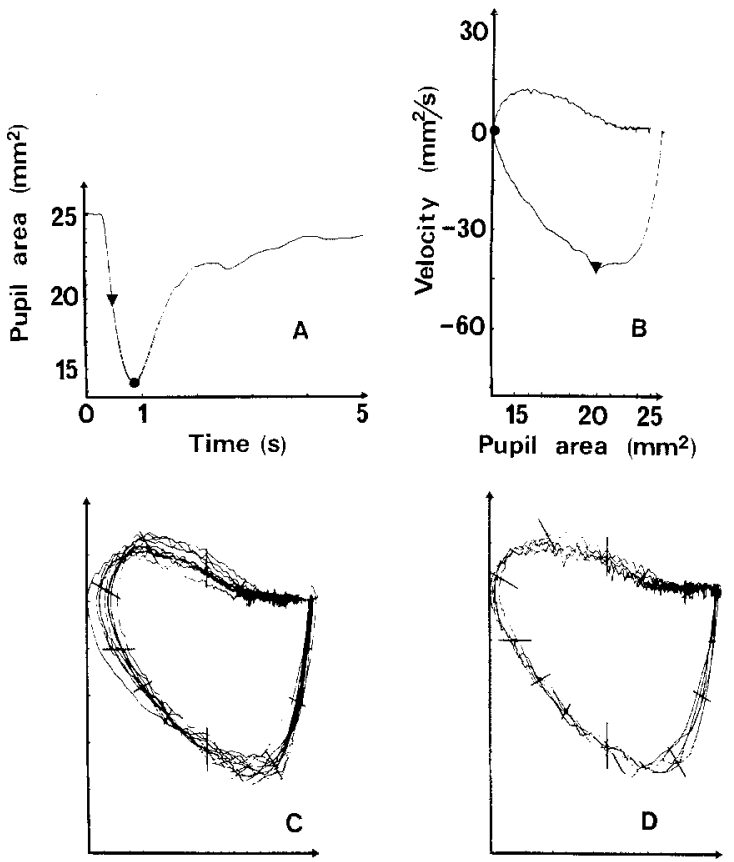

Fig. 7. A Light response to a $256 \mathrm{~ms}$ square wave stimulus in a control subject. B Plot of simultaneous values of response velocity $\left(\mathrm{mm}^{2} / \mathrm{s}\right)$ and size during the response seen in $\mathbf{A}$. The lower part of the curve corresponds to the $\sim 0.9$ s constriction phase, the upper part is the remaining $\sim 5 \mathrm{~s}$ redilatation phase. The black $\operatorname{dot}(\boldsymbol{)})$ on the curve in $\mathbf{A}$ and $\mathbf{B}$ corresponds to the minimum size of the pupil during the response and the arrow ( $\boldsymbol{\nabla}$ ) denotes the direction of the response. C The size-velocity plots of the light responses in 11 control subjects. Each plot is translated to a common origin and is further redrawn isomorphically to enclose a fixed area. The bars indicate mean $\pm 2 \mathrm{SD}$ with respect to 12 directions from an arbitrary centre. D The size-velocity plot in those five diabetic patients who had the smallest pupils, transformed as for the control subjects $C$ and with mean $\pm S D$ of the controls superimposed for direct comparison. The complexity of shape makes it impossible to test statistically any difference, but it is evident that these most severely affected diabetic patients do not show at any time during the response any systematic deviation from the shape of the velocity-size plot in control subjects

There was no difference in latency time or constriction time between the diabetic and control subjects (Table 2) and neither of these parameters was correlated to the duration of diabetes (Fig. 5).

The maximal constriction velocity was reduced in the diabetic patients (Table 2), but it was closely related to the response amplitude, and this relationship was identical in both groups ( $r=0.91, p<0.000001$, Fig. 6). Likewise, the maximal redilatation velocity was less in diabetic patients than in control subjects (Table 2) and was closely correlated to the response amplitude in both groups (diabetic patients: $r=0.79, p<0.000001$, control subjects: $r=0.76, p<0.000001$, the regression lines being identical). The velocities of constriction and redilatation in the diabetic patients were also, though to a less pronounced degree, correlated with the initial pupil size $(r=0.50, p<0.000001$, and $r=0.55, p<0.000001)$. In the control subjects, however, there was no correlation between these parameters and initial pupil size. Constriction and redilatation velocities both showed an inverse 
relationship to the duration of diabetes $(r=-0.29$, $p=0.043$, and $r=-0.28, p=0.0073$, respectively).

The redilatation time tended to be higher in diabetic patients than control subjects (Table 2), but the difference was not significant, nor was there any difference between long-term diabetic patients and the control subjects. However, a significant correlation was obtained between redilatation time and the duration of diabetes ( $r=0.22, p=0.033$; data not shown).

Another measure of the redilatation phase, the relative redilatation residue, tended to be larger in the diabetic patients, but the difference was not significant.

The velocity size plots for 11 control subjects and five long-term diabetic patients (who had the smallest pupil areas) are shown in Figure 7.

As expected, the results of stimulation with the stronger light pulse of $50 \mu \mathrm{lm}$ showed a larger response amplitude, shorter latency time, greater velocities of contraction and redilatation, but unchanged constriction and redilatation time in both groups. Employing the stronger light pulse of $50 \mu \mathrm{lm}$, the same differences between diabetic patients and control subjects were obtained as with the weaker light pulse of $25 \mu \mathrm{lm}$.

\section{Discussion}

The present study of the light reflex of the pupil in diabetic patients was performed on a much larger group of patients than previously reported $[9,10,12]$. Of even more importance is the fact that: (1) these patients were young (aged 25-42 years), (2) their duration of diabetes ranged from 0 to 32 years, independent of age, (3) only patients with a normal or near-normal retina in the area stimulated by the light were included and (4) the technique employed assured equality of the effective stimulus. The many parameters derived were obtained by computerized calculations.

The finding of a smaller initial or resting pupil size in diabetic patients confirms earlier reports [11, 16-19]. We have demonstrated previously that the abnormally small pupil in diabetes is correlated with the degree of long-term metabolic control and to peripheral neuropathy, retinopathy and nephropathy [11].

The response amplitude was smaller in diabetic patients than in control subjects, as also found in previous studies. However, an abnormally small pupil of, e.g. $10 \mathrm{~mm}^{2}$, cannot be expected to constrict as much (e.g. $13 \mathrm{~mm}^{2}$ ) to a given light stimulus as a normal and larger pupil of $36 \mathrm{~mm}^{2}$ (Fig. 1). This simple fact raises the question: what is a normal response for an abnormally small pupil? At first sight, it seems reasonable to consider the response of the pupil to light as related to its size at rest, before the stimulus was applied. It is well known that the retina is a highly adaptive organ, i. e. it is very insensitive to even tenfold differences in intensity of constant light. Thus, being almost without any information about the absolute illumination and the absolute pupil size, the retina mainly senses the relative pupil response to a sudden change in its illumination.

However, this simple way of regarding the relationship between pupil size and reaction to light in a given subject is broken down among normal subjects by the inverse correlation between pupil size and relative response amplitude (Fig.4). Among diabetic patients, we found the same inverse correlation, although this does not help in further understanding the problem.

There is, however, one peculiarity that must, in some way, be related to diabetic autonomic neuropathy: for a given pupil size in the normal range, the diabetic subjects had a slightly smaller relative response amplitude, probably indicating some damage to the efferent, parasympathetic pathway.

That the situation in the patients whose pupils are definitely smaller than normal, defies analysis is illustrated by a recent study [20] where 40-year-old diabetic patients were compared with 60 -year-old control subjects.

It has long been known that rubeosis iridis is a feature of diabetic microangiopathy $[8,21]$. One may therefore consider that a vascular abnormality of the iris, leading to its relative 'stiffness' could invalidate the results obtained in our study.

However, the velocity-size plot, to a certain degree, argues against this. Irrespective of the stimulus intensity, its shape is constant in any given subject independent of the variations in initial pupil size, e.g. when produced by stimuli repeated in quick succession [22]. Varying stimulus modalities, on the other hand, produce markedly varying shapes of the velocity-size plot. Thus, we would conclude that the absence of any detectable abnormalities in the shape of the velocity-size plot excludes the possibility of a major change in the dynamic properties of the pupil. The identity in diabetic patients and control subjects of the same intimate relationship between maximal velocities of constriction and redilatation and the response amplitude is further indirect evidence against severe changes in the iris structure as the cause of the small response. The present results are in agreement with an earlier study in which rhythmic light stimulation of the pupil with increasing frequencies revealed no evidence for abnormal stiffness, even in the smallest pupil in diabetic patients [19]. It is certainly conceivable that patients with advanced diabetic iridopathy with extensive neovascularization of the iris $[8,21]$ would have a diminished pupillary response. However, this long-term diabetic manifestation is very rare [23] and only two of our 66 diabetic patients, who underwent slit lamp examination, had rubeosis iridis, and only to a minute degree. These two patients and the eight patients with porosity of the pigment seam and loss of pigment had a pupillary response that was similar to the other diabetic patients.

Most previous reports have shown prolonged latency time in diabetic patients, but in none was the conclusion likely to be uninfluenced by the omission of some 
of the methodological and clinical details underlined above. In particular, unless proper algorithms are used, a large variation in constriction velocities and amplitudes will tend to produce a spurious inverse relationship between response amplitudes and latency times (Fig. 6), as demonstrated previously [17].

A recent study of the light response in newly diagnosed diabetic patients revealed no changes during poor metabolic control except for delayed redilatation [24]. On the other hand, the light-induced unrest in the pupil and the ability to maintain miosis under continuous illumination were markedly diminished.

Recently, it has been demonstrated that the small pupil in diabetes is associated with supersensitivity to sympathomimetic agents [25, 26]. Most evidence therefore suggests the small pupil size to be a result of damage to the sympathetic nerves, which, in turn, is a part of diabetic autonomic neuropathy leaving the parasympathetic innervated sphincter pupillae to constrict the pupil. As previously mentioned, Ohrt [8] found a prolonged redilatation in a number of diabetic patients and suspected this to be the result of sympathetic nervous dysfunction.

The tendency to prolonged redilatation shown in the present study would also support a sympathetic defect. That the pupil is still capable of reacting normally within its altered dynamic range also indicates that the parasympathetic innervation to the pupillary sphincter is relatively less affected.

Acknowledgements. We are indebted to Professor K. Lundbæk for his untiring support and for performing the ophthalmoscopic and biomicroscopic examinations in the majority of the patients, and to Professor N. Ehlers, Department of Ophthalmology, University of Aarhus, for permission to use ophthalmological data on the remaining patients. The secretarial assistance of A. B. Andersen, A. Larsen and M.V. Kristjánsdóttir is gratefully acknowledged. This study was supported by the Danish Medical Research Council and the Icelandic Science Foundation.

\section{References}

1. Pryce TD (1887) Perforating ulcers of both feet associated with diabetes and ataxic symptoms. Lancet $2: 11-12$

2. Pitres MA (1902) Note sur l'état des réflexes cutanés et pupillaires er des sensibilités testiculaire et épigastrique profondes chez les diabetiques. CR Soc Biol 4: 1286-1287

3. Major RH (1924) Tabes diabetica. JAMA 83: 2004

4. Waite JH, Beetham WP (1935) The visual mechanism in diabetes mellitus. A comparative study of 2002 diabetics, and 457 non-diabetics for control. N Engl J Med 212: 367-379

5. Jordan WR (1936) Neuritic manifestations in diabetes mellitus. Arch Intern Med 57: 307-366

6. Rundles RW (1945) Diabetic neuropathy. General review with report of 125 cases. Medicine 24: 111-160

7. Martin MM (1953) Diabetic neuropathy. A clinical study of 150 cases. Brain $76: 594-624$
8. Ohrt V (1967) Diabetic iridopathy. Thesis. Universitetsforlaget, Aarhus

9. Friedman SA, Feinberg R, Podolak E, Bedell RHS (1967) Pupillary abnormalities in diabetic neuropathy. A preliminary study. Ann Intern Med 67: 977-983

10. Gliem H (1971) Pupillomotorische Veränderungen bei Diabetikern. Acta Ophthalmol (Kbh) 49:955-963

11. Hreidarsson ÁB (1982) Pupil size in insulin-dependent diabetes, relationship to duration, metabolic control, and long-term manifestations. Diabetes 31: 442-448

12. Gundersen HJG (1976) Nervous and muscular dynamics of the pupil in long-term diabetics. In: Bajaj JS (ed) Current topics in diabetes research. IX Congress of the International Diabetes Federation. Excerpta Medica, Amsterdam, p 131-132

13. Steiness I (1957) Vibratory perception in diabetics. Acta Med Scand 158: $327-335$

14. Gundersen HJG (1976) A new photostimulator and videopupillograph for quantitative neuroophthalmological studies. Ophthalmologica 172: 62-68

15. Stark L (1968) Neurological control systems. Studies in bioengineering. Plenum, New York

16. Gundersen HJG (1974) An abnormality of the central autonomic nervous system in long-term diabetes: absence of hippus. Diabetologia 10: 366 (Abstract)

17. Smith SE, Smith SA, Brown PM, Fox C, Sönksen PH (1978) Pupillary signs in diabetic autonomic neuropathy. Br Med J 4: 924-927

18. Pfeifer MA, Cook D, Brodsky J, Tice D, Parrish D, Reenan A, Halter JB, Porte Jr D (1982) Quantitative evaluation of sympathetic and parasympathetic control of iris function. Diabetes Care 5: $518-528$

19. Hreidarsson ÁB (1979) Pupil motility in long-term diabetes. Diabetologia 17: 145-150

20. Smith SA, Smith SE (1983) Reduced pupillary light reflexes in diabetic autonomic neuropathy. Diabetologia 24: 330-332

21. Gartner S, Henkind P (1978) Neovascularization of the iris (Rubeosis Iridis). Surv Ophthalmol 22: 291-312

22. Semmlow J, Stark L (1973) Pupil movements to light and accommodative stimulation: a comparative study. Vision Res 13: $1087-1100$

23. Lundbæk K (1953) Long-term diabetes. The clinical picture in diabetes mellitus after 15-25 years duration, with a follow-up of a regional series of cases. (The ophthalmological section in collaboration with VA Jensen). Munksgaard, Copenhagen

24. Hreidarsson ÁB (1981) Acute, reversible autonomic nervous system abnormalities in juvenile insulin-dependent diabetes. Diabetologia 20: 475-481

25. Hayashi M, Ishikawa S (1979) Pharmacology of pupillary responses in diabetics - correlative study of the responses and grade of retinopathy. Jap J Ophthalmol 23: 65-72

26. Smith SA, Smith SE (1983) Evidence for a neuropathic aetiology in the small pupil of diabetes mellitus. Br J Ophthalmol 67: 89-93

Received: 3 July 1984

and in revised form: 17 August 1985

Dr. Ástrádur B. Hreidarsson

Medical Department

Landspítalinn

University Hospital

Reykjavik

Iceland 